\title{
NANOPARTICLES RESHAPE THE BIOMEDICAL INDUSTRY
}

\author{
Parichehr Hassanzadeh $^{1 *}$, Fatemeh Atyabi ${ }^{\mathbf{1}, 2}$, and Rassoul Dinarvand ${ }^{\mathbf{1 , 2}}$ \\ ${ }^{1}$ Nanotechnology Research Center, Faculty of Pharmacy, Tehran University of Medical Sciences, Tehran, Iran \\ ${ }^{2}$ Department of Pharmaceutics, Faculty of Pharmacy, Tehran University of Medical Sciences, Teheran, Iran
}

Over the last decades, increasing interest has been attracted towards the nanotechnology which provide a set of promising research tools and theranostic approaches. Tremendous research efforts in nanofabrication technology have led to the production of biocompatible nanostructures and advanced carriers with various configurations for protection of the loaded biomolecules or drugs against the metabolism or excretion. Furthermore, controlled delivery and targeted therapy may result in the improved therapeutic effects against a variety of diseases and reduced adverse effects of drugs. The efficiency of protein drugs may be negatively affected by their limited transportation within the body and short half-lives. Application of nanoparticles may significantly improve the pharmacological profiles of protein drugs. In neurology, high-resolution imaging techniques, nanoengineered materials capable of interaction with the nervous systems, and nanopharmaceuticals with minimal toxicity and improved bioavailability may be of great theranostic significance. This may provide remarkable breakthroughs in the pharmaceutical industry and health-care system. In the present review, the significance of nanotechnology and modeling approaches in health-care system has been highlighted. Biomed Rev 2018; 29: 17-26

Keywords: nanomedicine, drug delivery, nanopharmaceuticals

\section{INTRODUCTION}

Enormous research efforts in nanotechnology and application of the highly-advanced nanodevices with various configurations have provided attractive research tools and theranostic approaches against a variety of disorders. High-resolution imaging may provide deeper understanding about the pathomechanisms of disorders leading to the selection of more appropriate treatment strategies and obtaining better treatment outcomes. Real time detection of a variety of biomarkers and molecular signals or using the nanoscale-targeted magnetic resonance imaging (MRI) contrast agents demonstrate the immense potential of nanomaterials in the early diagnostics and prognostics $(1,2)$. Development of the biocompatible nanomaterials for protection of therapeutics against the metabolism or excretion and overcoming the chemo-resistance

Received 24 November 2018, revised 2 December 2018, accepted 3 December 2018.

* Correspondence to: Parichehr Hassanzadeh, Nanotechnology Research Center, Faculty of Pharmacy, Tehran University of Medical Sciences, Tehran, Iran Tel.: +98 21 66959095; Fax: +98 21 66581558; Cell phone: +989121887745 E-mail: p-hassanzadeh@razi.tums.ac.ir 
might result in the improved treatment outcome. In this context, designing the nanopharmaceuticals with improved halflives and bioavailability and targeted drug delivery systems using a variety of nanoparticles including the polymer- or lipid-based ones has attracted a growing interest. The ability of nanocarriers for improving the pharmacokinetics and in vivo stability of oligonucleotides, peptides, and proteins has also been well-documented (3-7). Limited efficiency of the conventional treatment strategies against the neurological diseases has resulted in designing the new treatment options such as the development of nanodevices capable of interaction with the nervous systems or multifunctional nanoparticles for delivery of therapeutics across the blood-brain barrier (BBB) for improving their bioavailability and reducing adverse effects (8). Nanotechnology along with tissue engineering are promising approaches for designing the controlled release systems containing the bioactive proteins or a variety of drugs which may re-establish the functional neuronal networks or neuronal connectivity (9-11). Carbon nanotubes (CNTs) with superior mechanical properties, improved biocompatibility, and high thermo-electrical conductivities, have shown enormous potential in a variety of scientific fields. Besides application for biosensing, non-invasive and high-resolution imaging, CNTs may be applied as the carriers of therapeutics against a variety of disorders (12-18). Early detection of neoplastic or precancerous lesions has remained challenging that may be partly due to the insufficiency of spatial resolution of cancer imaging technologies for early detection. Development of nanotech-based imaging technologies enables to provide better definition for anatomical aspects of lesions, identify the molecular expressions of neoplasms, and detect the early-stage tumors (19). In recent years, increasing research efforts have been attracted towards the development of novel nanobiosensors and nanotherapeutics for cancer theranostics (20-22). Nanocantilever arrays and nanowires may be applied for early detection of malignant lesions that may be of paramount importance in cancer therapy (23). Using nanocantilever, nanowire, and nanotube arrays facilitates the transition from single to multiple biomarker detection and selection of more appropriate strategies for cancer therapy (24). Nanoparticles may also be applied for biomarker detection ex vivo. For optical identification of the leukaemia cells in blood samples, fluorophore-laden silica beads have been successfully used (25). Furthermore, quantum dot bio-conjugates and fluorescent nanoparticles may be used for recognition of the molecular signatures and ultrasensitive detection of DNA, respectively
(26). In prostate cancer, lymph-node metastases may not be detectable by traditional approaches, however, application of the lymphotropic paramagnetic nanoparticles may be helpful in this regard (27). Bimodal nanoparticles as the carriers of near-infrared optically detectable fluorochrome, have been conjugated to MRI contrast agent leading to the efficient brain tumor imaging and intra-operative visualization of the lesions (28). In mice, gadolinium-loaded polymeric dendrimers have been successfully applied for imaging the lymphatic of breast cancer (29). Nanoparticle probes which are targeted with recognition agents enable to obtain valuable information relating the distribution or abundance of cancer markers (30). Moreover, angiogenesis has been successfully imaged in the animals receiving targeted nanoparticle formulations (31). These all indicate the key significance of nanotechnology in development of the next generation of therapeutics.

\section{NANOVECTORS}

These nanostructures are usually applied for non-invasive visualization of the molecular markers or targeted delivery of therapeutics. Nanovectors protect the active agents against the enzymatic degradation, reduce the clearance time of peptide drugs, and overcome the biological barriers. Multifunctionality and delivery of large amounts of imaging agents or therapeutics in a tissue-specific fashion are the major advantages of nanovectors. Using the biological targeting moieties enables to achieve a wide variety of new therapeutics. Several polymer-based nanovectors have been represented for application in the clinical settings $(32,33)$. For systemic administration of the nanovectors, silica and silicon may be applied $(34,35)$. Furthermore, metal-based nanovectors may be applied for localized thermal ablation that might be of significance in eradication of tumors (36). Molecular targeting of the active agent-loaded nanovectors may be achieved via conjugation with recognition moieties leading to the enhanced specificity. Advantages of the molecularly-targeted nanovectors over traditional antibody-guided treatment strategies include the capability of carrying various targeting agents, delivery of greater therapeutic payloads, co-localized delivery of multiple active agents, and bypassing the biological barriers (37). Indeed, barrier-avoiding nanovectors may provide remarkable advances in designing more efficient anticancer agents with fewer adverse effects. Attaching fluorescein to the nanovectors provides the optical-imaging signals that might be promising in targeted cancer therapies (38). Tumor-targeting mechanism or enhanced permeation and retention (EPR) is a 
key issue which should be taken into account in development of the novel formulations of nanovectors (39). In this sense, application of the appropriate mathematical formulations may be useful for designing the nanovectors with optimized EPR (40). Co-administration of a bradykinin antagonist has been shown to increase the endothelial vascular permeability that may result in the enhanced EPR targeting of nanovectors (41). As follows, the widely-applied nanoparticle systems for biomedical applications including the polymeric and lipid nanoparticles have been highlighted.

\section{POLYMERIC NANOPARTICLES}

Over the last decade, a variety of polymers have been developed for preparation of nanoparticles as the solid nanovectors which are usually prepared using the single materials. Polymeric nanoparticles with high loading capacity appear to be more stable, easy-to-prepare, and safer than other nanocarriers. Nonimmunogenicity and rapid elimination from the body make the selected polymers as suitable candidates for development of nanoparticles with tunable surface properties and capable of encapsulating a variety of therapeutics. Selecting the proper polymeric carriers facilitates the controlled release of loaded drugs $(42,43)$. Besides the significance of nanoparticle size, scalability, biodegradability, and biocompatibility, appropriate linker design is of key importance for obtaining the optimal treatment outcome (44). Polymeric nanoparticles containing interleukin-10 have been shown to reduce the inflammation in atherosclerotic plaques. This type of nanoparticles have been represented as suitable alternatives to traditional anti-inflammatory agents (45). Polymers may also be applied for delivery of water-soluble proteins and bio-imaging. Near-infrared dye loaded polymeric nanoparticles have been applied for cancer imaging and treatment (46). Using polyethyleneimine for preparation of biocompatible and cell-permeable polymeric nanoparticles has provided higher fluorescence which may be useful for visualizing caspase-dependent apoptosis (47).

Using the surface modification techniques, polymeric nanoparticles may be applied for delivery of proteins, nucleic acids, or small molecule drugs to the central nervous system (CNS). For drug delivery into the CNS, various synthetic or natural polymers including the polyesters, poly(methylidene malonates), poly(alkylcyanoacrylates), or polysaccharides may be applied for preparation of the nanoparticles that may result in promising therapeutic effects against the neurodegenerative disorders or malignancies (48-50). In recurrent gliomas, chemotherapeutic-loaded polymer nanoparticles capable of controlled drug delivery have improved the survival of patients (51). For treatment of the intracranial tumors, convectionenhanced delivery (CED) of drug-loaded polymer nanoparticles has been suggested as a useful approach which may transport the nanoparticles over the clinically relevant volumes of distribution $(52,53)$. Poly(lactic-co-glycolic acid) (PLGA) nanoparticles loaded with camptothecin have been delivered via CED, an effective approach against the intracranial tumor model (54). Because of the attractiveness of CED, this strategy has been applied in the randomized clinical trials for delivery of larger amounts of therapeutics without any significant systemic toxicity (55). Meanwhile, intracranial CED may be impractical for a number of patients due to its invasivity. In this respect, controlling the size, surface coatings, and charge of polymer nanoparticles should be taken into consideration for more effective drug delivery (56). Microspheres have been known as the first particulate systems for direct drug delivery towards the brain. Polymeric microspheres fabricated from a wide variety of materials such as chitosan, poly(methylidene malonate), poly(epsilon-caprolactone), or PLGA, may be applied for local delivery of various therapeutic agents against the malignant gliomas $(57,58)$. Noteworthy, delivery of the polymeric nanoparticles into the CNS largely depends on the adsorptive- or receptor-mediated transcytosis using targeting ligands or cell-penetrating peptides. Poly(butylcyanoacrylate) (PBCA) nanoparticles have been known as the first polymerbased nanocarriers for drug delivery into the CNS (59). Polysorbate 80 , a commonly used surfactant, increases the CNS penetration of PBCA nanoparticles that may be due to the modulation of efflux transporters or tight junctions, interaction with endothelial receptors of $\mathrm{BBB}$, or reducing the clearance of nanoparticles $(60,61)$. Coating of PLGA nanoparticles with polysorbate 80 may also increase the delivery efficiency of drug-loaded nanoparticles across the BBB (62). Following the systemic administration, PLGA-, poly(glycolic acid) (PGA)-, or poly(lactic acid) (PLA)-based nanoparticles may penetrate the BBB in a size-dependent fashion (63). In order to bypass the efflux transporters, trans-activating transcriptor peptide has been attached to the surface of drug-loaded PLA nanoparticles leading to the enhanced transport of nanoparticles into the CNS (64). Chitosan is a naturally occurring biodegradable and biocompatible polysaccharide which may be applied for development of nanocarriers for efficient delivery of therapeutics across the BBB (65). Following the systemic administration, chitosan nanoparticles capable of delivery of dopamine, caspase inhibitors, or peptides, have provided 
promising neuroprotective effects (66). Moreover, cationic polymers may be applied for development of nanoparticles for delivery of nucleic acids. Surface modification of this type of nanoparticles with rabies virus glycoprotein-derived peptides enables the transvascular delivery of small interfering RNA to the CNS (67).

\section{LIPID NANOPARTICLES}

Lipid-based colloidal drug delivery systems including the nanostructured lipid carriers (NLCs) and solid lipid nanoparticles (SLNs) have been designed as the alternatives of polymeric nanoparticles, liposomes, or emulsions. This type of nanoparticles capable of targeted drug delivery and protecting the loaded therapeutics against the enzymatic degradation, improve the bioavailability of therapeutics and their efficiency (68). Because of the limitations of SLNs including the drug expulsion during the storage and limited drug loading capacity (69), NLCs (the mixture of liquid and solid lipids) with longterm stability, suitable biocompatibility, high drug loading capacity, and controlled drug release profile that may result in the reduced drug dosage and side effects, are usually preferred for encapsulating of poorly water-soluble therapeutics (70). Because of the biocompatibility of excipients and aqueous nature, NLCs are suitable for intravenous drug delivery (71).

Application of NLCs in cancer chemotherapy may result in the improved therapeutic index of loaded anti-cancer agents (72). Besides application for tumor targeting, NLCs as sustained-release drug carrier systems have been represented as promising nanoformulations against the ischemic neural injuries, fungal infections, and leukemia (73-78).

Atorvastatin, a widely applied lipid-regulating drug, is associated with poor aqueous solubility and first pass metabolism that may negatively affect the efficiency of drug. In this respect, designing the drug delivery systems capable of improving drug solubility and bypassing the hepatic metabolism might be of therapeutic importance. Development of atorvastatin-loaded NLCs by high-speed homogenization and ultrasonication has improved the pharmacological profile of the drug indicating the appropriateness of NLC formulations (79). As compared to the commercially available tacrolimus ointment, tacrolimus-loaded NLCs have shown enhanced skin penetration (80). Regarding the inhalation drug delivery, NLCs have shown longer residence time in lungs than liposomes, emulsions, or polymeric nanoparticles and greater stability against the shear forces created during the nebulization that may be due to their lipophilic character and small particle size. This make NLCs suitable for loading drugs against the pulmonary disorders (81). Furthermore, providing sustained drug release and prolonged residence time for the entrapped lipophilic drugs represent NLCs as promising nanocarriers for ocular drug delivery (82). Using NLCs, it may also be possible to increase the stability and bioavailability of drugs against the cardiovascular disorders and prolong their circulation time that may result in the improved treatment outcome (83).

\section{APPLICATION OF MODELING APPROACHES: THE NECESSITY OF NANOMEDICINE}

Despite the innovative techniques in nanotechnology which may be applied for development of stable nanoparticulate systems capable of high loading capacity and targeted delivery of theranostics, data reliability, personalized theranostics, or occurrence of the unexpected phenomena appear as challenging issues (84). Modeling and simulation techniques may be applied in order to design safer nanoparticles with appropriate bioactivity and selectivity (85). Multi-scale modeling techniques enable to develop shape-specific nanoparticles and assess their functional characteristics in vivo. It may also be possible to evaluate the effects of surface properties, shape, and size of nanoparticles on drug loading, release profiles, or cellular uptake and predict nanoparticle behavior in the biological systems (86). Moreover, the cause(s) of failure in targeted drug delivery may be analyzed leading to the development of more efficient nanoformulations (87). Modeling of the adhesion kinetics, transportation, and cellular internalization of nanoparticles may reduce the non-specific sequestration and increase their bioavailability (88). Furthermore, using the suitable algorithms enables to calculate the binding probability of nanoparticles with specific typed of receptors (89). Ultrafine nanoneedles which are thinner than the smallest single-walled carbon nanotubes, may be applied for transferring proteins, genes, or ions into the cells. Application of the computational quantum chemistry enables to develop stable structures with highly-symmetrical geometry (90). For mimicking tumor microenvironment, nanoparticles may be incubated in co-culture systems that may facilitate designing the effective drug delivery systems. Application of the modeling techniques for identification of the key parameters which affect the targeting specificity and cellular uptake of nanoparticles or determining the co-culture ratios in the heterogeneous populations of cells might be of great significance (91). Noteworthy, the pharmacological profiles of drugs including their efficiency or toxicity may 
be affected by the genetic backgrounds (92). Patient-specific models may provide opportunities for personalized treatment or diagnostics. Furthermore, prediction of drug efficiency, resistance, adverse effects, or treatment response as well as pharmacogenomic analysis may be possible using the suitable modeling techniques (93). For personalized nanoparticle drug delivery into the blood vessels, isogeometric analysis may be applied for simulating the vascular permeability, binding, and transport of nanoparticles. Appropriate modeling approaches enable to assess the hydrodynamic conditions, vascular geometry, or surface density of the endothelial receptors (94). Besides providing a deeper knowledge about the pathomechanism of CNS diseases, modeling approaches enable tracking the real-time dynamics of receptors, evaluation of the information processing, signaling cascades, dynamics of synaptic connectivity, and performance of nano-engineered devices or multifunctional nanoparticles capable of neuronal stimulation $(95,96)$. Modeling methods may also be of key importance for designing the nanobiosensors or nanorobots with optimal function and configuration for biomedical applications (97-100). It appears that modeling approaches along with nanointerventions would dramatically affect the health-care system.

\section{CONCLUSION}

Selective drug delivery to the target tissues or organs which results in the improved bioavailability and reduced side effects of therapeutics, has been a challenging issue. Nanotechnology, an attractive interdisciplinary topic by which a wide variety of highly-advanced devices or biomaterials may be developed for early disease diagnosis or targeted therapy, has opened up a novel frontier in biomedicine. Nanotech-based research efforts have provided remarkable growth in designing more efficient delivery systems capable of overcoming the biological barriers and providing improved treatment outcomes. Using nanotechnology tools enables to obtain deeper knowledge about the pathomechanisms of a variety of disorders leading to the development of more effective therapeutics. Designing the nanopharmaceuticals with minimal toxicity and improved bioavailability as well as the biomaterials capable of interaction with biological systems might be of great theranostic significance. Multiscale modeling, high-performance simulation techniques, and patient-specific models along with nano-interventions may significantly affect the development strategies of the next generation therapeutics.

\section{CONFLICT OF INTEREST}

The authors declare no conflict of interest.

\section{REFERENCES}

1. Heta Y, Kumaki K, Hifumi H, Citterio D, Tanimoto A, Suzuki K. Gadolinium containing photochromic micelles as potential magnetic resonance imaging traceable drug carriers. Photochem Photobiol 2012; 88: 876-883. DOI: 10.1111/j.1751-1097.2012.01124.x.

2. Kurakhmaeva KB, Djindjikhashvili IA, Petrov VE, Balabanyan VU, Voronina TA, Trofimov SS, et al. Brain targeting of nerve growth factor or using poly(butyl cyanoacrylate) nanoparticles. J Drug Target 2009; 17: 564-574. DOI: 10.1080/10611860903112842.

3. Patel T, Zhou J, Piepmeier JM, Saltzman WM. Polymeric nanoparticles for drug delivery to the central nervous system. Adv Drug Deliv Rev 2012; 64: 701-705. DOI: 10.1016/j.addr.2011.12.006.

4. Qiu L, Zheng C, Jin Y, Zhu K. Polymeric micelles as nanocarriers for drug delivery. Expert Opin Ther Pat 2007; 17: 819-830. DOI:10.1517/13543776.17.7.819.

5. Sun NF, Meng QY, Tian AL, Hu SY, Wang RH, Liu ZX, et al. Nanoliposome mediated FL/TRAIL double-gene therapy for colon cancer: in vitro and in vivo evaluation. Cancer Lett 2012; 315: 69-77. DOI:10.1016/j.canlet. 2011.10.010.

6. Hassanzadeh P, Atyabi F, Dinarvand R. Nanoencapsulation: A Promising strategy for biomedical application of ferulic acid. Biomed Rev 2017; 28: 26-34. DOI: http:// dx.doi.org/10.14748/bmr.v28.4449.

7. Hassanzadeh P, Arbabi E, Rostami F, Atyabi F, Dinarvand R. Aerosol delivery of ferulic acid-loaded nanostructured lipid carriers: A promising treatment approach against the respiratory disorders. Physiol Pharmacol 2017; 21: 331-342. http://phypha.ir/ppj/ article-1-1295-en.html.

8. Hassanzadeh P. Nanopharmaceuticals: Innovative theranostics for the neurological disorders. Biomed Rev 2014; 25: 25-34. DOI: 10.14748/bmr.v25.1043.

9. Fuchs JR, Nasseri BA, Vacanti JP. Tissue engineering: a 21st century solution to surgical reconstruction. Ann Thorac Surg 2001; 72: 577-591. PMID:11515900.

10. Hassanzadeh P. Tissue engineering and growth factors: updated evidence. Biomed Rev 2012; 23: 19-35.

11. Hassanzadeh P, Atyabi F, Dinarvand R. Tissue engineering: Still facing a long way ahead. J Control Release 2018; 279: 181-197. DOI:10.1016/j.jconrel.2018.04.024 
12. Jan E, Kotov NA. Successful differentiation of mouse neural stem cells on layer by layer assembled single walled carbon nanotube composite. Nano Lett 2007; 7 : 1123-1128. DOI: 10.1021/n10620132.

13. Hassanzadeh P, Atyabi F, Dinarvand R. Application of carbon nanotubes for controlled release of growth factors or endocannabinoids: A breakthrough in biomedicine. Biomed Rev 2016; 27: 19-27. DOI: 10.14748/bmr. v27.2105.

14. Hassanzadeh P, Arbabi E, Atyabi F, Dinarvand R. Nerve growth factor-carbon nanotube complex exerts prolonged protective effects in an in vitro model of ischemic stroke. Life Sci 2017; 179: 15-22. DOI: 10.1016/j. lfs.2016.11.029.

15. Hassanzadeh P, Arbabi E, Atyabi F, Dinarvand R. Application of carbon nanotubes as the carriers of the cannabinoid, 2- arachidonoylglycerol: Towards a novel treatment strategy in colitis. Life Sci 2017; 179: 66-72. DOI:10.1016/j.lfs.2016.11.015.

16. Hassanzadeh P, Arbabi E, Rostami F, Atyabi F, Dinarvand R. Carbon nanotubes prolong the regulatory action of nerve growth factor on the endocannabinoid signaling. Physiol Pharmacol 2015; 19: 167-176. http://phypha.ir/ ppj/article-1-1112-en.html.

17. Hassanzadeh P, Arbabi E, Atyabi F, Dinarvand R. Carbon nanotubes provide longer lasting gastroprotective effects for anandamide in stress-induced gastric ulcer in rat. Physiol Pharmacol 2018; 22: 38-47. http://phypha. ir/ppj/article-1-1266-en.html.

18. Hassanzadeh P, Arbabi E, Atyabi F, Dinarvand R. Carbon nanotube-anandamide complex exhibits sustained protective effects in an in vitro model of stroke. Physiol Pharmacol 2016; 20: 12-23. http://phypha.ir/ppj/article1-1155-en.html.

19. Morawski AM, Winter PM, Crowder KC, Caruthers SD, Fuhrhop RW, Scott MJ, et al. Targeted nanoparticles for quantitative imaging of sparse molecular epitopes with MRI. Magn Reson Med 2004; 51: 480-486. DOI:10.1002/ mrm.20010.

20. Hassanzadeh P. New perspectives in biosensor technology. Gastroenterol Hepatol Bed Bench 2010; 3: 105-107.

21. Hassanzadeh P, Fullwood I, Sothi S, Aldulaimi D. Cancer nanotechnology. Gastroenterol Hepatol Bed Bench 2011; 4: 63-69. PMID: 24834159.

22. Shi J, Kantoff PW, Wooster R, Farokhzad OC. Cancer nanomedicine: progress, challenges and opportunities.
Nat Rev 2017; 17: 20-37. DOI:10.1038/nrc.2016.108.

23. Cui Y, Qingqiao W, Hongkun P, Lieber CM. Nanowire nanosensors for highly sensitive and selective detection of biological and chemical species. Science 2001; 293: 1289-1292. DOI: 10.1126/science.1062711.

24. Su M, Li S, Dravid V. Microcantilever resonance-based DNA detection with nanoparticle probes. Appl Phys Lett 2003; 82: 3562-3564. DOI:10.1063/1.1576915

25. Santra S, Tan W. Conjugation of biomolecules with luminophore-doped silica nanoparticles for photostable biomarkers. Anal Chem 2001; 73: 4988-4993. https:// pubs.acs.org/doi/abs/10.1021/ac010406.

26. Zhao X, Tan W. Ultrasensitive DNA detection using highly fluorescent bioconjugated nanoparticles. $J \mathrm{Am}$ Chem Soc 12003; 25: 11474-11475. DOI: 10.1021/ ja0358854.

27. Harishingani MG, , Barentsz J, Hahn PF, Deserno WM, Tabatabaei $\mathrm{S}$, van de $\mathrm{Kaa} \mathrm{CH}$, et al. Noninvasive detection of clinically occult lymph-node metastases in prostate cancer. N Engl J Med 2003; 348: 2491-2499. DOI:10.1056/NEJMoa022749.

28. Kircher MF, Mahmood U, King RS, Weissleder R, Josephson L. A multimodal nanoparticle for preoperative magnetic resonance imaging and intraoperative optical brain tumor delineation. Cancer Res 2003; 63: 81228125.PMID: 14678964.

29. Park JW. Liposome-based drug delivery in breast cancer treatment. Breast Cancer Res 2002; 4: 95-99. https:// www.ncbi.nlm.nih.gov/pubmed/12052251.

30. Li KCP, Pandit SD, Guccione S, Bednarski MD. Molecular imaging applications in nanomedicine. Biomed Microdevices 2004; 6: 113-116. DOI:10.1023/ B:BMMD.0000031747.05317.81.

31. Winter PM, Wickline SA, Lanza GM. Molecular imaging of angiogenesis in nascent $\mathrm{Vx}-2$ rabbit tumors using a novel $\alpha_{\mathrm{v}} \beta_{3}$-targeted nanoparticle and 1.5 tesla magnetic resonance imaging. Cancer Res 2003; 63: 5838-5843. https://www.ncbi.nlm.nih.gov/pubmed/14522907.

32. Duncan R. The dawning era of polymer therapeutics. Nature Rev Drug Discov 2003; 2: 347-360. DOI:10.1038/ $\operatorname{nrd} 1088$.

33. Gilles EM, Frechet JMJ. Designing macromolecules for therapeutic applications: Polyester dendrimerpolyethylene oxide 'bow-tie' hybrids with tunable molecular weights and architecture. J Am Chem Soc 2002; 124: 14137-14146. PMID:12440912. 
34. Yan F, Kopelman R. The embedding of metatetra( hydroxyphenyl)-chlorin into silica nanoparticle platforms for photodynamic therapy and their singlet oxygen production and $\mathrm{pH}$-dependent optical properties. Photochem Photobiol 2003; 78: 587-591. PMID:14743867.

35. Cohen MH, Melnik K, Boiasrki A, Ferrari M, Martin FJ. Microfabrication of silicon-based nanoporous particulates for medical applications. Biomed Microdevices 2003; 5: 253-259.DOI: DOI:10.1023/A:1025768411300.

36. Hirsch LR, Halas NJ, West JL. Nanoshell-mediated near-infrared thermal therapy of tumors under magnetic resonance guidance. Proc Natl Acad Sci USA 2003; 100: 13549-13554. https://www.ncbi.nlm.nih.gov/pubmed/14597719.

37. Decuzzi P, Lee S, Decuzzi M, Ferrari M. Adhesion of micro-fabricated particles on vascular endothelium: a parametric analysis. Ann Biomed Eng 2004; 32: 793-802. https://www.ncbi.nlm.nih.gov/pubmed/15255210.

38. Quintana A, Baker JN Jr. Design and function of a dendrimer-based therapeutic nanodevice targeted to tumor cells through the folate receptor. Pharm Res 2002; 19: 1310-1316. https://www.ncbi.nlm.nih.gov/ pubmed/12403067.

39. Jain RK. Delivery of molecular and cellular medicine to solid tumors. Adv Drug Deliv Rev 2001; 46: 149-168. https://www.ncbi.nlm.nih.gov/pubmed/11259838.

40. Decuzzi P, Lee S, Bhushan B, Ferrari M. A theoretical model for the margination of particles within blood vessels. Ann Biomed Eng 2005; 33: 179-190. DOI:10.1007/ s10439-005-8976-5.

41. Wu J, Akaka T, Maeda H. Modulation of enhanced permeability in tumor by a bradykinine antagonist, a cyclooxygenase inhibitor. Cancer Res 1998; 58: 159-165. https://www.ncbi.nlm.nih.gov/pubmed/9426072.

42. Chen G, Lu J, Lam C, Yu Y. A novel green synthesis approach for polymer nanocomposites decorated with silver nanoparticles and their antibacterial activity. Analyst 2014; 139: 5793-5799. https://www.ncbi.nlm.nih.gov/ pubmed/25199560.

43. Cheng LC, Chen HM, Lai TC, Chan YC, Liu RS, Sung $\mathrm{JC}$, et al. Targeting polymeric fluorescent nanodiamondgold/silver multi-functional nanoparticles as a lighttransforming hyperthermia reagent for cancer cells. Nanoscale 2013; 5: 3931-3940. https://www.ncbi.nlm. nih.gov/pubmed/23536050.
44. Hassanzadeh P, Atyabi F, Dinarvand R. Linkers: The key elements for the creation of efficient nanotherapeutics. J Control Release 2018; 270: 260-267. DOI:10.1016/j. jconrel.2017.12.007

45. Demento SL, Eisenbart SC, Foellmer HG, Platt C, Caplan MJ, Saltzman WM, et al. Inflammasome-activating nanoparticles as modular systems for optimizing vaccine efficacy. Vaccine 2009; 27: 3013-3021.https://www.ncbi. nlm.nih.gov/pubmed/19428913.

46. Lei T, Fernandez-Fernandez A, Manchanda R, Huang YC, McGoron AJ. Near-infrared dye loaded polymeric nanoparticles for cancer imaging and therapy and cellular response after laser-induced heating. Beilstein $\mathrm{J} \mathrm{Na}$ notechnol 2014; 5: 313-322. DOI:10.3762/bjnano.5.35.

47. Kim K, Lee M, Park H, Kim JH, Kim S, Chung H, et al. Cell-permeable and biocompatible polymeric nanoparticles for apoptosis imaging. J Am Chem Soc 2006; 128: 3490-3491. DOI: 10.1021/ja057712f.

48. Li SY, Wang M. Hybrid polymer-metal nanospheres based on highly branched nanoparticles for potential medical applications. IET Nanobiotechnol 2012; 6: 136-143. DOI: 10.1049/iet-nbt.2011.0050.

49. Woodrow KA, Cu Y, Booth CJ, Saucier-Sawyer JK, Wood MJ, Saltzman WM. Intravaginal gene silencing using biodegradable polymer nanoparticles densely loaded with small-interfering RNA. Nat Materials 2009; 8: 526-533. https://www.ncbi.nlm.nih.gov/pubmed/19404239.

50. Jacobs A, Voges J, Reszka R, Lercher M, Gossmann A, Kracht L, et al. Positron-emission tomography of vectormediated gene expression in gene therapy for gliomas. Lancet 2001; 358: 727-729.

51. Brem H, Piantadosi S, Burger PC, Walker M, Selker R, Vick NA, et al. Placebo-controlled trial of safety and efficacy of intraoperative controlled delivery by biodegradable polymers of chemotherapy for recurrent gliomas. Lancet 1995; 345: 1008-1012.

52. Chen MY, Hoffer A, Morrison PF, Hamilton JF, Hughes J, Schlageter KS, et al. Surface properties, more than size, limiting convective distribution of virus-sized particles and viruses in the central nervous system. J Neurosurgery 2005; 103: 311-319.

53. Sawyer AJ, Saucier-Sawyer JK, Booth CJ, Liu J, Patel $\mathrm{T}$, Piepmeier JM, et al. Convection-enhanced delivery of camptothecin-loaded polymer nanoparticles for treatment of intracranial tumors. Drug Deliv Trans Res 2011; 1: 34-42. [PubMed:21691426]. 
54. Saucier-Sawyer JK, Seo YE, Gaudin A, Quijano E, Song E, Sawyer AJ, et al. Distribution of polymer nanoparticles by convection-enhanced delivery to brain tumors. J Control Release 2016; 232: 103-112. DOI: 10.1016/j. jconrel.2016.04.006.

55. Kunwar S, Chang S, Westphal M, Vogelbaum M, Sampson J, Barnett G, et al. Phase III randomized trial of CED of IL13-PE38QQR vs Gliadel wafers for recurrent glioblastoma. Neurooncology 2010; 12: 871-881. https:// www.ncbi.nlm.nih.gov/pubmed/20511192.

56. Grodzinski D. Nanoparticle Trojan horses gallop from the lab into the clinic. Science 2010; 330: 314-315. DOI: 10.1126/science.330.6002.314.

57. Fung LK, Shin M, Tyler B, Brem H, Saltzman WM. Chemotherapeutic drugs released from polymers: distribution of 1,3-bis(2-chloroethyl)-1-nitrosourea in the rat brain. Pharm Res 1996; 13: 671-682.

58. Yemisci M, Bozdag S, Cetin M, Soylemezoglu F, Capan Y, Dalkara T, et al. Treatment of malignant gliomas with mitoxantrone-loaded poly (lactide-co-glycolide) microspheres. Neurosurgery 2006; 59: 1296-1302.

59. Kreuter J, Alyautdin RN, Kharkevich DA, Ivanov AA. Passage of peptides through the blood brain barrier with colloidal polymer particles (nanoparticles). Brain Res 1995; 674: 171-174.

60. Kreuter J, Shamenkov D, Petrov V, Ramge P, Cychutek $\mathrm{K}$, Koch-Brandt C, et al. Apolipoprotein-mediated transport of nanoparticle-bound drugs across the blood-brain barrier. J Drug Target 2002; 10: 317-325. [PubMed: 12164380].

61. Schroeder U, Schroeder H, Sabel BA. Body distribution of $3 \mathrm{H}$-labelled dalargin bound to poly(butyl cyanoacrylate) nanoparticles after i.v. injections to mice. Life Sci 2000; 66: 495-502. [PubMed: 10794066].

62. Kulkarni SA, Feng SS. Effects of surface modification on delivery efficiency of biodegradable nanoparticles across the blood-brain barrier. Nanomedicine 2011; 6: 377-394. [PubMed: 21385139].

63. Liu M, Li H, Luo G, Liu Q, Wang Y. Pharmacokinetics and biodistribution of surface modification polymeric nanoparticles. Arch Pharm Res 2008; 31: 547-554. [PubMed:18449515].

64. Rao KS, Reddy MK, Horning JL, Labhasetwar V. TATconjugated nanoparticles for the CNS delivery of antiHIV drugs. Biomaterials 2008; 29: 4429-4438. [PubMed: 18760470].
65. Songjiang Z, Lixiang W. Amyloid-beta associated with chitosan nano-carrier has favorable immunogenicity and permeates the BBB. AAPS Pharm Sci Tech 2009; 10: 900-905. [PubMed:19609682].

66. Karatas H, Aktas Y, Gursoy-Ozdemir Y, Bodur E, Yemisci M, Caban S, et al. A nanomedicine transports a peptide caspase- 3 inhibitor across the blood-brain barrier and provides neuroprotection. J Neurosci 2009; 29: 13761-13769. [PubMed: 19889988].

67. Kumar P, Wu H, McBride JL, Jung KE, Hee Kim M, Davidson BL, et al. Transvascular delivery of small interfering RNA to the central nervous system. Nature 2007; 448: 39-43. [PubMed: 17572664].

68. Fang JY, Fang CL, Liu CH, Su YH. Lipid nanoparticles as a vehicles for topical psoralen delivery: Solid lipid nanoparticles (SLN) versus nanostructured lipid carriers (NLC). Eur J Pharm Biopharm 2008; 70: 633-640. DOI: 10.1016/j.ejpb.2008.05.008.

69. Müller RH, Maeder K, Gohla S. Solid lipid nanoparticles (SLN) for controlled drug delivery. A review of the state of the art. Eur J Pharm Biopharm 2000; 50: 161-177. DOI:10.1016/S0939-6411(00)00087-4.

70. Puri A, Loomis K, Smith B, Lee JH, Yavlovich A, Heldman E, et al. Lipid-based nanoparticles as pharmaceutical drug carriers: From concepts to clinic. Crit Rev Ther Drug Carrier Syst 2009; 26: 523-580. PMID: 20402623.

71. Shi F, Yang G, Guo T, Du Y, Ren NF. Formulation design, preparation, and in vitro and in vivo characterizations of $\beta$-Elemene-loaded nanostructured lipid carriers. Int $J$ Nanomedicine 2013; 8: 2533-2541. https://www.ncbi. nlm.nih.gov/pubmed/23901271.

72. Sai Li, Zhigui Su, Minjie Sun, Yanyu Xiao, Feng Cao, Aiwen Huang, et al. An arginine derivative contained nanostructure lipid carriers with $\mathrm{pH}$-sensitive membranolytic capability for lysosomolytic anti-cancer drug delivery. Int J Pharm 2012; 436: 248-257. https://www. ncbi.nlm.nih.gov/pubmed/22732672.

73. Shenoy VS, Vijay IK, Murthy RS. Tumour targeting: biological factors and formulation advances in injectable lipid nanoparticles. J Pharm Pharmacol 2005; 57: 411421. https://www.ncbi.nlm.nih.gov/pubmed/15831200.

74. Hassanzadeh P, Arbabi E, Atyabi F, Dinarvand R. Ferulic acid-loaded nanostructured lipid carriers: A promising nanoformulation against the ischemic neural injuries. Life Sci 2018; 193: 64-76. DOI:10.1016/j. lfs.2017.11.046. 
75. Hassanzadeh P, Atyabi F, Dinarvand R. Dehpour AR, Azhdarzadeh M, Dinarvand M. Application of nanostructured lipid carriers: the prolonged protective effects for sesamol in in vitro and in vivo models of ischemic stroke via activation of PI3K signalling pathway. DARU J Pharm Sci 2017; 25: 1-16. DOI 10.1186/s40199-0170191-z.

76. Mendes AI, Silva AC, Catita JAM, Cerqueira F, Gabriel C, Lopes CM. Miconazole-loaded nanostructured lipid carriers (NLC) for local delivery to the oral mucosa: Improving antifungal activity. Colloids Surf B: Biointerfaces 2013; 111: 755- 763. DOI: 10.1016/j. colsurfb.2013.05.041.

77. Rahman HS, Rasedee HS, How CW, Abdul AB, Zeenathul NA, Othman HH, et al. Zerumbone-loaded nanostructured lipid carriers: preparation, characterization, and antileukemic effect. Int J Nanomed 2013; 8: 2769-2781. DOI: $10.2147 /$ IJN.S54346.

78. Bondì ML, Azzolina A, Craparo EF, Botto C, Amore E, Giammona G, et al. Entrapment of an EGFR inhibitor into nanostructured lipid carriers (NLC) improves its antitumor activity against human hepatocarcinoma cells. J Nanobiotech 2014; 12: 1-9. DOI: 10.1186/1477-315512-21.

79. Elmowafy M, Ibrahim HM, Ahmed MA, Shalaby K, Salama A, Hefesha H. Atorvastatin-loaded nanostructured lipid carriers (NLCs): strategy to overcome oral delivery drawbacks. Drug Deliv 2017; 24: 932-941. DOI:10.108 0/10717544.2017.1337823.

80. Nam SH, Ying X, Park JS. Investigation of Tacrolimus loaded nanostructered lipid carrier for topical drug delivery. Bull Korean Chem Soc 2011; 32: 956-960. DOI: 10.5012/bkcs.2011.32.3.956.

81. Patlolla RR, Chougule M, Patel AR, Jackson T, Tata PN, Singh M. Formulation, characterization and pulmonary deposition of nebulized celecoxib encapsulated nanostructured lipid carriers. J Control Release 2010; 144: 233-241. https://www.ncbi.nlm.nih.gov/pub$\mathrm{med} / 20153385$.

82. Araujo JS, Nikolic MA, Egea EB, Garcia ML. Nanostructured lipid carriers for triamcinolone acetonide delivery to the posterior segment of the eye. Colloids Surf B Biointerfaces 2011; 88: 150-157. https://www.ncbi.nlm. nih.gov/pubmed/21764568.

83. Zhanga WL, Gua X, Baib H, Yang RH, Donga CD, Liu JP. Nanostructured lipid carriers constituted from high-den- sity lipoprotein components for delivery of a lipophilic cardiovascular drug. Int J Pharm 2010; 391: 313-321. https://www.ncbi.nlm.nih.gov/pubmed/20214958.

84. Roukes M. Plenty of room, indeed. Sci Am 2001; 285: 54 57. https://www.ncbi.nlm.nih.gov/pubmed/11524969.

85. Chiesa S, de la Iglesia D, Crespo J, Martin-Sanchez F, Kern J, Potamias G, et al. European efforts in nanoinformatics research applied to nanomedicine. Stud Health Technol Inform 2009; 150: 757-761. PMID:19745412.

86. Shah S, Liu Y, Hu W, Gao J. Modeling particle shapedependent dynamics in nanomedicine. J Nanosci Nanotechnol 2011; 11:919-928. DOI: 10.1166/jnn.2011.3536.

87. Lehtinen J, Magarkar A, Stepniewski M, Hakola S, Bergman M, Róg T, et al. Analysis of cause of failure of new targeting peptide in PEGylated liposome: molecular modeling as rational design tool for nanomedicine. Eur J Pharm Sci 2012; 4: 121-130. DOI:10.1016/j. ejps.2012.02.009.

88. Gentile F, Ferrari M, Decuzzi P. The transport of nanoparticles in blood vessels: the effect of vessel permeability and blood rheology. Ann Biomed Eng 2008; 36: 254-261. DOI: 10.1007/s10439-007-9423-6.

89. Lee TR, Chang YS, Choi JB, Liu WK, Kim YJ. Numerical simulation of a nanoparticle focusing lens in a microfluidic channel by using immersed finite element method. J Nanosci Nanotechnol 2009; 9: 7407-7411. PMID:19908798.

90. Poater A, Gallegos Saliner A, Carbo-Dorca R, Poater J, Solà M, Cavallo L, et al. Modeling the structure-property relationships of nanoneedles: a journey toward nanomedicine. Comput Chem 2009; 30: 275-284. DOI: 10.1002/ jcc. 21041.

91. Costa EC, Gaspar VM, Marques JG, Coutinho P, Correia IJ. Evaluation of nanoparticle uptake in coculture cancer models. PLoS One 2013; 8: e70072. DOI: 10.1371/journal.pone.0070072.

92. Evans WE, Mcleod HL. Pharmacogenomics - drug disposition, drug targets, and side effects. N Engl J Med 2003; 348: 538-549. DOI:10.1056/NEJMra020526.

93. Ghosh S, Matsuoka Y, Asai Y, Hsin KY. Software for systems biology: from tools to integrated platforms. Nat Rev Genet 2011; 12: 821-832. DOI: 10.1038/nrg3096.

94. Hossain SS, Zhang Y, Liang X, Hussain F, Ferrari M, Hughes TJ, et al. In silico vascular modeling for personalized nanoparticle delivery. Nanomedicine 2013; 8: 343-357. DOI:10.2217/nnm.12.124. 
95. Kotaleski JH, Blackwell KT. Modelling the molecular mechanisms of synaptic plasticity using systems biology approaches. Nat Rev Neurosci 2010; 11: 239-251. DOI: 10.1038/nrn2807.

96. Schoeberl B, Eichler-Jonsson C, Gilles ED, Müller G. Computational modeling of the dynamics of the MAP kinase cascade activated by surface and internalized EGF receptors. Nat Biotechnol 2002; 20: 370-375. DOI:10.1038/nbt0402-370.

97. Hassanzadeh P. Computational modelling: Moonlighting on the neuroscience and medicine. Biomed Rev 2013; 24: 25-31. DOI: 10.14748/bmr.v24.19.
98. Hassanzadeh P, Atyabi F, Dinarvand R. Application of modelling and nanotechnology-based approaches: The emergence of breakthroughs in theranostics of central nervous system disorders. Life Sci 2017; 182: 93-103. DOI:10.1016/j.lfs.2017.06.001.

99. Hassanzadeh P, Atyabi F, Dinarvand R. Ignoring the modeling approaches: Towards the shadowy paths in nanomedicine. J Control Release 2018; 280: 58-75. DOI:10.1016/j.jconrel.2018.04.042.

100. Hassanzadeh P, Atyabi F, Dinarvand R. Creation of nanorobots: Both state-of-the-science and state-of-the-art. Biomed Rev 2016; 27: 37-44. 